OPEN ACCESS

Edited by:

Yizhang Jiang,

Jiangnan University, China

Reviewed by:

Thomas Bolton,

Advanced Telecommunications

Research Institute International (ATR),

Japan

Ling-Li Zeng,

National University of Defense

Technology, China

Linling Li,

Shenzhen University, China

*Correspondence:

Fengchun Wu

13580380071@163.com

Kai Wu

kaiwu@scut.edu.cn

Specialty section:

This article was submitted to

Brain Imaging Methods,

a section of the journal

Frontiers in Neuroscience

Received: 29 September 2021 Accepted: 01 December 2021

Published: 11 January 2022

Citation:

Wang J, Ke P, Zang J, Wu F and

Wu K (2022) Discriminative Analysis of Schizophrenia Patients Using

Topological Properties of Structural

and Functional Brain Networks:

A Multimodal Magnetic Resonance

Imaging Study.

Front. Neurosci. 15:785595.

doi: 10.3389/fnins.2021.785595

\section{Discriminative Analysis of} Schizophrenia Patients Using Topological Properties of Structural and Functional Brain Networks: A Multimodal Magnetic Resonance Imaging Study

\author{
Jing Wang', Pengfei Ke',6, Jinyu Zang',6, Fengchun Wu ${ }^{3,4 *}$ and Kai $W u^{2,3,4,5,6,7,8,9 *}$ \\ ${ }^{1}$ School of Biomedical Engineering, Guangzhou Xinhua University, Guangzhou, China, ${ }^{2}$ School of Biomedical Sciences \\ and Engineering, South China University of Technology, Guangzhou, China, ${ }^{3}$ The Affiliated Brain Hospital of Guangzhou \\ Medical University, Guangzhou, China, ${ }^{4}$ Guangdong Engineering Technology Research Center for Translational Medicine \\ of Mental Disorders, Guangzhou, China, ${ }^{5}$ Guangdong Engineering Technology Research Center for Diagnosis \\ and Rehabilitation of Dementia, Guangzhou, China, ${ }^{6}$ National Engineering Research Center for Tissue Restoration \\ and Reconstruction, South China University of Technology, Guangzhou, China, ${ }^{7}$ Key Laboratory of Biomedical Engineering \\ of Guangdong Province, South China University of Technology, Guangzhou, China, ${ }^{8}$ Institute for Healthcare Artificial \\ Intelligence Application, Guangdong Second Provincial General Hospital, Guangzhou, China, ${ }^{9}$ Department of Nuclear \\ Medicine and Radiology, Institute of Development, Aging and Cancer, Tohoku University, Sendai, Japan
}

Interest in the application of machine learning $(\mathrm{ML})$ techniques to multimodal magnetic resonance imaging (MRI) data for the diagnosis of schizophrenia (SZ) at the individual level is growing. However, a few studies have applied the features of structural and functional brain networks derived from multimodal MRI data to the discriminative analysis of SZ patients at different clinical stages. In this study, 205 normal controls (NCs), 61 first-episode drug-naive SZ (FESZ) patients, and 79 chronic SZ (CSZ) patients were recruited. We acquired their structural MRI, diffusion tensor imaging, and restingstate functional MRI data and constructed brain networks for each participant, including the gray matter network (GMN), white matter network (WMN), and functional brain network (FBN). We then calculated 3 nodal properties for each brain network, including degree centrality, nodal efficiency, and betweenness centrality. Two classifications (SZ vs. NC and FESZ vs. CSZ) were performed using five ML algorithms. We found that the SVM classifier with the input features of the combination of nodal properties of both the GMN and FBN achieved the best performance to discriminate SZ patients from NCs [accuracy, 81.2\%; area under the receiver operating characteristic curve (AUC), 85.2\%; $p<0.05$ ]. Moreover, the SVM classifier with the input features of the combination of the nodal properties of both the GMN and WMN achieved the best performance to discriminate FESZ from CSZ patients (accuracy, 86.2\%; AUC, 92.3\%; $p<0.05)$. Furthermore, the brain areas in the subcortical/cerebellum network and the 
frontoparietal network showed significant importance in both classifications. Together, our findings provide new insights to understand the neuropathology of SZ and further highlight the potential advantages of multimodal network properties for identifying SZ patients at different clinical stages.

Keywords: schizophrenia, brain networks, discriminative analysis, machine learning, multimodal MRI

\section{INTRODUCTION}

Schizophrenia (SZ) is a chronic psychiatric disease with hallucinations, delusions, and cognitive dysfunction ( $\mathrm{Lu}$ et al., 2016). With the development of magnetic resonance imaging (MRI), the vast majority of studies have shown structural and functional brain abnormalities in SZ patients (Yamasue et al., 2004; Antonova et al., 2005; Kuroki, 2006; Pagsberg et al., 2007; Zhou et al., 2007). Several structural MRI studies have reported SZ patients show hippocampal volume reduction and bilateral thalamus volume reduction compared with normal controls (NCs) (Adriano et al., 2010, 2012), whereas the most reported functional alterations in SZ patients are located in thalamus, medial frontal gyrus, and superior temporal gyrus (Ren et al., 2013; Turner, 2013). Furthermore, previous studies have indicated that structural brain abnormalities are more widespread in chronic SZ (CSZ) patients than in first-episode drug-naive SZ (FESZ) patients, suggesting the potential impact of antipsychotic medication on structural brain abnormalities (Moncrieff and Leo, 2010; Torres et al., 2016). On the other hand, numerous functional MRI studies indicated that CSZ patients showed significant reductions in functional characteristics in brain regions involved in auditory, visual processing, and sensorimotor functions compared with FESZ patients (Wu et al., 2018). Therefore, it is critical to develop neuroimaging-based biomarkers for distinguishing the illness stages of SZ patients.

A number of studies based on the quantitative analysis of brain networks have reported that SZ patients show significantly decreased connectivity between a range of brain regions, particularly involving connections among the frontal lobe, temporal lobe, and insula compared with NCs (Bassett et al., 2008; Palaniyappan et al., 2015; Erdeniz et al., 2017). Previous studies of gray matter networks (GMNs) have shown that SZ patients exhibit reduced betweenness centrality (BC) in several regions and increased $\mathrm{BC}$ mainly in primary cortex and paralimbic cortex regions (Zhang et al., 2012). Numerous studies of white matter networks (WMNs) have demonstrated that a decreased clustering coefficient (Zhou et al., 2007; Collin et al., 2014), decreased global efficiency (van den Heuvel et al., 2013; Drakesmith et al., 2015; Griffa et al., 2015), and decreased node efficiency (NE) of the frontal lobe and limbic system were found in SZ patients (van den Heuvel et al., 2010; Wang et al., 2012; Sun et al., 2015). In addition, previous studies of functional brain networks (FBNs) indicated that the degree centrality (DC) in SZ patients was decreased in the bilateral putamen and increased in the left superior frontal gyrus (Chen et al., 2015). However, a few studies compared FESZ with CSZ patients based on the quantitative analysis of brain networks.
Recently, machine learning (ML) methods using neuroimaging data have been increasingly applied in the classification between SZ patients and normal controls (NCs), in which the classification accuracy varies from 0.65 to 0.95 (Arbabshirani et al., 2013; Talpalaru et al., 2019; Cao et al., 2020; Guo et al., 2020; Steardo et al., 2020). The majority of previous studies have mainly applied ML methods to a single neuroimaging modality, including structural MRI (sMRI) (Xiao et al., 2017; Oh et al., 2020), diffusion tensor imaging (DTI) (Ingalhalikar et al., 2010; Ardekani et al., 2011), resting-state functional MRI (rs-fMRI) (Koch et al., 2015; Skåtun et al., 2017; Cai et al., 2020), and electroencephalogram (EEG) (Ke et al., 2021). More recently, some studies have used multimodal MRI data to detect $\mathrm{SZ}$ at the level of the individual, and most measures are derived from multimodal MRI features, such as gray matter volume, regional homogeneity, amplitude of low-frequency fluctuation, and degree of centrality (Lu et al., 2018; Liang et al., 2019; Lei D. et al., 2020). Our previous study has applied multimodal MRI features to distinguish the illness stages of SZ patients based on ML methods and the findings have contributed to stage-specific biomarkers in diagnosis and interventions of SZ (Wu et al., 2018).

The objective of this study is to apply the features of both structural and functional brain networks derived from multimodal MRI data to the discriminative analysis of SZ patients at different clinical stages. We constructed three types of brain networks, including GMN, WMN, and FBN, for each participant derived from sMRI, DTI and rs-fMRI data. Three nodal properties of each brain networks were calculated, including BC, DC, and NE. The performance of two classifications (SZ vs. NC and FESZ vs. CSZ) was analyzed by using different combinations of nodal properties as the input features. Five ML algorithms were applied in both discriminative analyses, including the linear support vector machine (SVM) (Cortes, 1995), random forest algorithm (RF) (Breiman, 1996), logistic regression (LR) (Peng et al., 2002), linear discriminant analysis (LDA) (Fisher, 1936), and K-nearest neighbor classification (KNN) (Zhang, 2016).

\section{MATERIALS AND METHODS}

\section{Subjects}

A total of 61 FESZ patients, 79 CSZ patients, and 205 NCs were recruited for this study. The SZ patients were diagnosed by trained and experienced clinical psychiatrists using a structured clinical interview according to Diagnostic and Statistical Manual of Mental Disorders: Fourth Edition, Text Revision (DSM-IV-TR) (Structured Clinical Interview for DSM 
Disorders [SCID]) criteria. The FESZ, CSZ, and NC groups were recruited from the Affiliated Brain Hospital of Guangzhou Medical University and the local community, respectively. All subjects were aged between 18 and 45 years, and their biological parents were Han Chinese. Before scanning, a clinical assessment was performed by psychiatrists using the Positive and Negative Syndrome Scale (PANSS) (Kay et al., 1987). The subjects obtained a consensus score for each item on all three subscales (positive symptoms, negative symptoms, and general psychopathology) that was based on a seven-point scale indicating the severity of the symptom (van Tol et al., 2014). The inclusion criteria for all SZ patients were as follows: (1) a total score of at least 60 for the three PANSS subscales and (2) at least 3 positive symptom items on the PANSS with a score of at least 4 . Additionally, FESZ patients were recruited for the first time when they were seeking help due to psychotic symptoms and did not take any antipsychotics. All CSZ patients were taking antipsychotics, and the course of the disease was greater than 2 years.

The exclusion criteria for all subjects included (1) any other psychiatric Axis I disorder meeting DSM-IV criteria, including schizoaffective disorders, intellectual disability, major depressive disorder, bipolar disorder, delirium, dementia, memory disorder, and other cognitive disorders; (2) mental disorder due to substance dependence, a seriously unstable somatic disease, definite diabetes, thyroid diseases, hypertension, or heart disease; (3) narrow angle glaucoma; (4) a history of epilepsy, except for febrile convulsions; (5) alcohol dependence meeting DSMIV-TR criteria (excluding nicotine dependence); (6) having received electroconvulsive therapy within the past 6 months; (7) a contraindication for MRI; (8) medical resource neuroleptic malignant syndrome or serious tardive dyskinesia; (9) a serious suicide attempt or an irritative state; (10) noncompliant drug administration or a lack of legal guardians; or (11) lactating, pregnant, planning to become pregnant. In addition, NCs were excluded if they had a first- or second-degree relative with a psychiatric Axis I disorder according to DSM-IV criteria. Before enrollment, all subjects or their legal guardians provided written informed consent. These studies were performed according to the Declaration of Helsinki and approved by the Ethics Committees of the Affiliated Brain Hospital of Guangzhou Medical University.

\section{Magnetic Resonance Imaging Data Acquisition}

MRI images were acquired using a Philips 3T MR system (Philips, Achieva, Netherlands) located at the Affiliated Brain Hospital of Guangzhou Medical University. The participants were instructed to keep their eyes closed, to relax but not fall asleep, and to move as little as possible. The sMRI data were obtained using a sagittal three-dimensional gradient-echo T1-weighted sequence $(256 \times 256 \times 188$ matrix with $1 \mathrm{~mm} \times 1 \mathrm{~mm} \times 1 \mathrm{~mm}$ spatial resolution, repetition time $(\mathrm{TR})=8.2 \mathrm{~ms}$, echo time $(\mathrm{TE})=3.8 \mathrm{~ms}$, flip angle $=7^{\circ}$, field of view $(\mathrm{FOV})=256 \mathrm{~mm} \times 256$ $\mathrm{mm})$. The DTI data were acquired using a single-shot echoplanar imaging-based sequence with the following parameters: slice thickness is $3 \mathrm{~mm}$, no gap, 50 axial slices, $\mathrm{TR}=6,000 \mathrm{~ms}$;
$\mathrm{TE}=70 \mathrm{~ms} ;$ flip angle $=90^{\circ} ; \mathrm{FOV}=256 \mathrm{~mm} \times 256 \mathrm{~mm}$; spatial resolution $=2 \mathrm{~mm} \times 2 \mathrm{~mm} \times 3 \mathrm{~mm} ; 33$ nonlinear diffusion weighting directions with $b=1000 \mathrm{~s} / \mathrm{mm}^{2}$ and one image without diffusion weighting $\left(b=0 \mathrm{~s} / \mathrm{mm}^{2}\right)$. The rs-fMRI data were collected using an echo-planar imaging (EPI) sequence $(64 \times 64 \times 36$ matrix with $3.44 \mathrm{~mm} \times 3.44 \mathrm{~mm} \times 4 \mathrm{~mm}$ spatial resolution, $\mathrm{TR}=2,000 \mathrm{~ms}, \mathrm{TE}=30 \mathrm{~ms}$, flip angle $=90^{\circ}$, $\mathrm{FOV}=220 \mathrm{~mm} \times 220 \mathrm{~mm})$.

\section{Image Processing}

All T1-weighted MRI data processing was performed using the SPM8 software package ${ }^{1}$ (Institute of Neurology, University College London, United Kingdom). First, each T1-weighted MRI was segmented into three tissue maps, including gray matter (GM), white matter (WM), and cerebrospinal fluid (CSF), using the new segmentation algorithm from SPM8. Second, a customized, population-specific template was created from the segmented tissue maps using the DARTEL template-creation tool. Third, all GM maps were warped to the custom template space using its corresponding smooth, reversible deformation parameters. A modulation was applied by locally multiplying tissue values by the Jacobian determinants derived from the special normalization step (Good et al., 2001). Spatial smoothing was not performed to avoid inducing artifactual signal overlap among spatially adjacent regions (Kong et al., 2021).

Preprocessing of the DTI dataset was implemented using $\mathrm{PANDA}^{2}$, which is a pipeline toolbox for diffusion MRI analysis (Cui et al., 2013). The procedure mainly included skull stripping, simple motion and eddy current correction, and diffusion tensor/parameter calculation. Eddy current was an important factor for image deformation, and affine transformation was used to register the DTI image to the T1 image, which can effectively reduce the influence of head movement and eddy current.

Rs-fMRI data were preprocessed using SPM8 (see text footnote 1) Institute of Neurology, University College London, United Kingdom) and DPABI (Yan et al., 2016). First, the first 10 volumes of each functional time series were discarded because the initial signal was unstable. Second, the remaining volumes were corrected for different signal acquisition times and realigned to the first volume to correct for head motion. Then, the nuisance signals (Rigid-body 6 motion parameters, the white matter signal, and the cerebrospinal fluid signal) were regressed out from the data. Subsequently, all functional volumes were normalized using EPI templates and resampled to $3-\mathrm{mm}$ isotropic voxels. The resampled data were bandpass $(0.01-0.08 \mathrm{~Hz})$ filtered to reduce low-frequency drift and high-frequency physiological noise and spatially smoothed with a 4-mm FWHM Gaussian kernel. All participants in the study did not have excessive head motion ( $<2 \mathrm{~mm}$ or $2^{\circ}$ during realignment and $<2 \mathrm{~mm}$ for mean motion during framewise assessment).

\section{Brain Network Construction}

The single-subject GMN for T1-weighted MRI data was constructed based on the GM volume images. A GMN here

\footnotetext{
${ }^{1}$ http://www.fil.ion.ucl.ac.uk/spm

${ }^{2}$ http://www.nitrc.org/projects/panda/
} 
included a collection of nodes and edges interconnecting the nodes. Here, the nodes represent brain regions, and edges represent interregional similarity in the distributions of regional GM volume. To define the network nodes, the GM volume image was parcellated into 268 regions of interest (ROIs) using a 268-node functional atlas (Shen et al., 2013). The GM volume value of each of the 268 ROIs was then calculated. We utilized a Kullback-Leibler (KL) divergence-based similarity (KLS) measure to estimate the network edges (Kong et al., 2014). Specifically, the probability density function of these 268 values was estimated using kernel density estimation (KDE) with bandwidths chosen automatically. The regional probability distribution function (PDF) and the KL divergence between each pair of 268 regions in their PDFs were then calculated. The network edges were defined as KLS with a consecutive sparsity threshold, $\mathrm{S}$, ranging from $0.1<\mathrm{S}<0.4$ (interval =0.01). All of the following network analyses were performed at each of the threshold levels in this range. For each of the network metrics, the estimated values under the range of 0.1-0.4 were integrated with area under curve (Wang et al., 2016).

The WMN for DTI data was also parcellated into 268 ROIs. According to deterministic white matter fiber bundle tractography, the fiber number (FN) connected in the ROIs was taken as the connection weight, and then the FN-weighted connection matrix was calculated. The parameters of fiber tractography were set: the turning angle between adjacent voxels was less than 45 degrees or fractional anisotropy was greater than 0.2 (Gong et al., 2009). The white matter connection was calculated using PANDA (Cui et al., 2013). The network edges were defined as the number of white matter fibers with the threshold FN $>2$.

The FBN for fMRI data was constructed based on the main regional time series by averaging voxelwise time series data. Pearson's correlation coefficient of the interregional time series was defined to measure the relation between network nodes. Then, each of the resulting correlation matrices was converted into a series of weighted networks with a sparsity threshold, S, ranging from $0.1<\mathrm{S}<0.4$ (interval $=0.01$ ). All of the following network analyses were performed at each of the threshold levels in this range. For each of the network metrics, the estimated values under the range of 0.1-0.4 were integrated with area under curve (Liu et al., 2017).

The nodal properties of each region, including DC, NE, and $\mathrm{BC}$, were then calculated using the GRETNA toolbox (Wang et al., 2015).

\section{Statistical Analysis}

Between-group differences in age and years of education were analyzed by one-way analysis of variance (ANOVA) using SPSS 22.0 software. A post hoc analysis was performed using Scheffés method. A $\chi^{2}$ test was performed to determine sex differences. Statistical significance was set at $p<0.05$.

\section{Discriminative Analysis}

In this study, we randomly split the set of participants into two groups, including a training dataset and an independent testing dataset, at a ratio of $4: 1$. In the training dataset, 10 -fold crossvalidation was performed. Each time, the ninefold data were used for training, and onefold data were used for validation. Each feature is normalized to between 0 and 1 on the training set, and the normalized parameters are applied to the validation set. Then, the performance of each resulting classifier was evaluated using the independent testing dataset. Using this method, we achieved unbiased estimates of every classifier.

The performance of two classifications (SZ patients vs. NC and FESZ patients vs. CSZ) was analyzed using $5 \mathrm{ML}$ algorithms, including SVM, RF, LR, LDA, and KNN. Moreover, we used the method of recursive feature elimination (RFE) (Lin et al., 2017) to iteratively remove redundant features while preserving discriminative features (Figure 1). The analyses for both classification tasks were performed by using the in-house software NEURO-LEARN ${ }^{3}$, which is a solution for collaborative pattern analysis of neuroimaging data (Lei B. et al., 2020).

From the results obtained by NEURO-LEARN, we obtained the weight of each input feature from the output of the classifier, and the absolute value of the feature weights can quantify the contribution of the features to the classifier. In this study, we calculated the feature contribution based on the results by the best classification performance. In addition, we divided 268 ROIs into eight subnetworks (Finn et al., 2015), including medial frontal, frontoparietal, default mode, subcortical/cerebellum, motor, visual I, visual II, and visual association (Figure 2). We selected the top 5\% of combined features (BC, DC, and $\mathrm{NE}$ ) ranked by the weights in the best classification to discuss subnetwork contributions.

To compare the performance of the $5 \mathrm{ML}$ algorithms, we plotted the receiver operating characteristic (ROC) curves and calculated the area under the ROC curve (AUC). A permutation test was applied to explore whether the AUC obtained by the proposed model was significantly higher than the AUC of a random guess by randomly permuting the labels of the training data 1,000 times prior to the training step followed by the entire classification process. Based on probability distributions, it is possible to test the null hypothesis. The statistical significance was set at $p<0.05$.

\section{RESULTS}

\section{Clinical and Demographic Characteristics}

The clinical and demographic characteristics of all subjects are shown in Table 1. There were significant differences in age and years of education were noted between NCs and CSZ patients $(p<0.05)$; there were also significant differences in age and years of education between NCs and FESZ patients $(p<0.05)$. Moreover, there was no significant difference in the positive, negative, general, and total PANSS scores between FESZ and CSZ patients.

\footnotetext{
${ }^{3}$ https://github.com/Raniac/NEURO-LEARN
} 


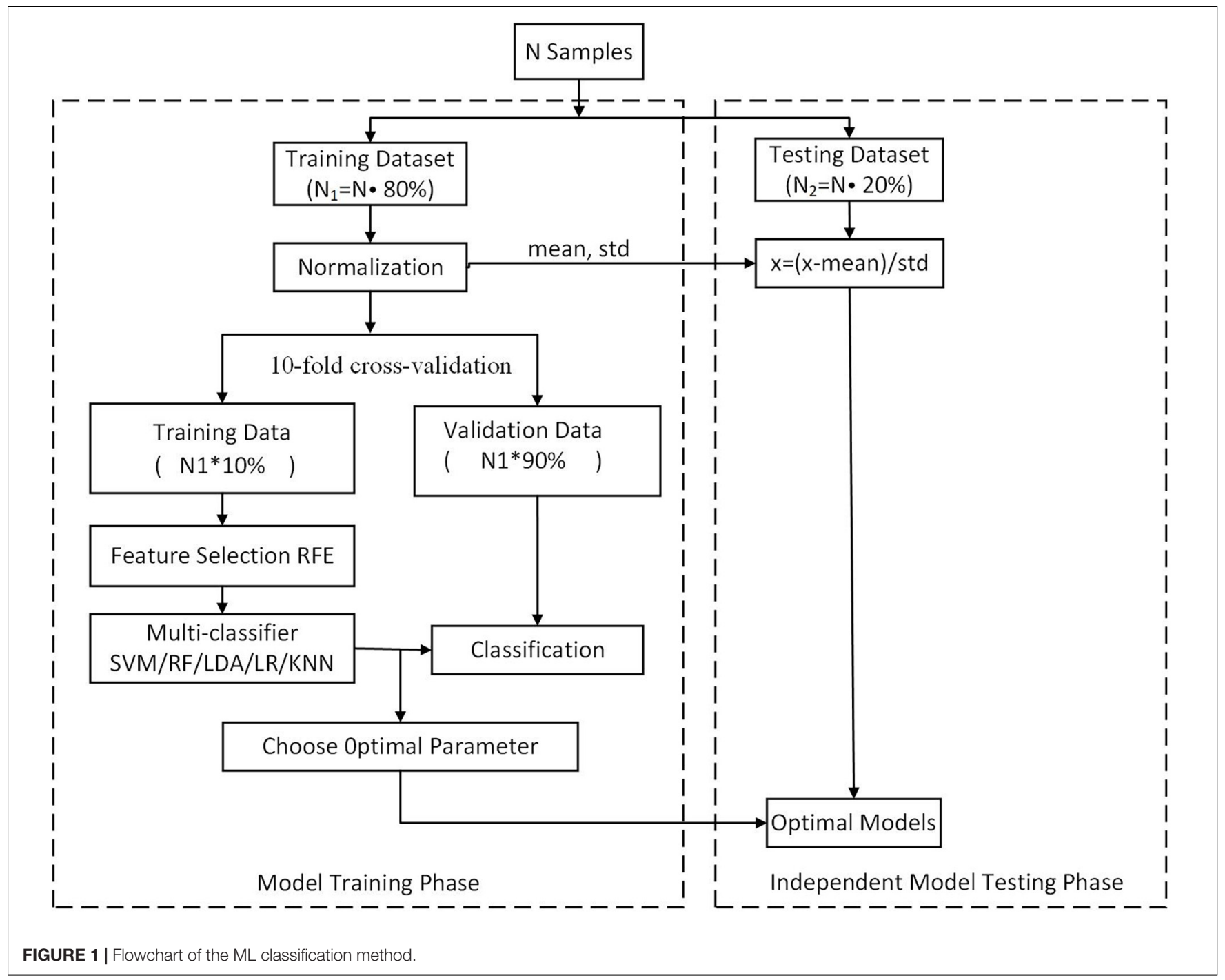

\section{Classification Performance}

Two classifications were performed to distinguish SZ patients from NCs as well as FESZ patients from CSZ patients. The network properties of GMN, WMN, and FBN were used as the input features of the five ML algorithms. The results indicated that the SVM achieved the best performance compared to the other four ML algorithms based on RFE in both classifications (Supplementary Tables 1, 2).

\section{Results of Classification Between Schizophrenia Patients and Normal Controls}

When discriminating SZ patients from NCs, the classifier of SVM with the input features of the properties of GMN and FBN achieved the highest performance with an accuracy of 81.2\% and AUC of 85.2\% $(p<0.05)$, as shown in Figure 3 and Supplementary Table 1.

We chose the top $5 \%$ of features ranked by their weights in the best classification (Supplementary Table 3). Meanwhile, we divided 268 ROIs into eight subnetworks and calculated the frequency of each subnetwork in the top 5\% of features from the GMN and FBN. We found that the ROIs from the GMN were distributed in the subcortical/cerebellum network, frontoparietal network, motor network, medial frontal network, visual I network, visual II network, and visual association network. The ROIs from the FBN were distributed in the subcortical/cerebellum network, frontoparietal network, medial frontal network, visual II network, visual association network, default mode network, and motor network (Figure 4).

Overall, the ROIs were mainly distributed in the subcortical/cerebellum network (30\%) and frontoparietal network (21.25\%), and the proportion of the features from the GMN was greater than the proportion of the features from the FBN in the classification between SZ patients and NCs.

\section{Results of Classification Between First-Episode Drug-Naive SZ and Chronic SZ Patients}

When discriminating FESZ from CSZ patients, the SVM classifier with the input features of the properties of GMN and WMN achieved the highest performance with an accuracy of $86.2 \%$ 

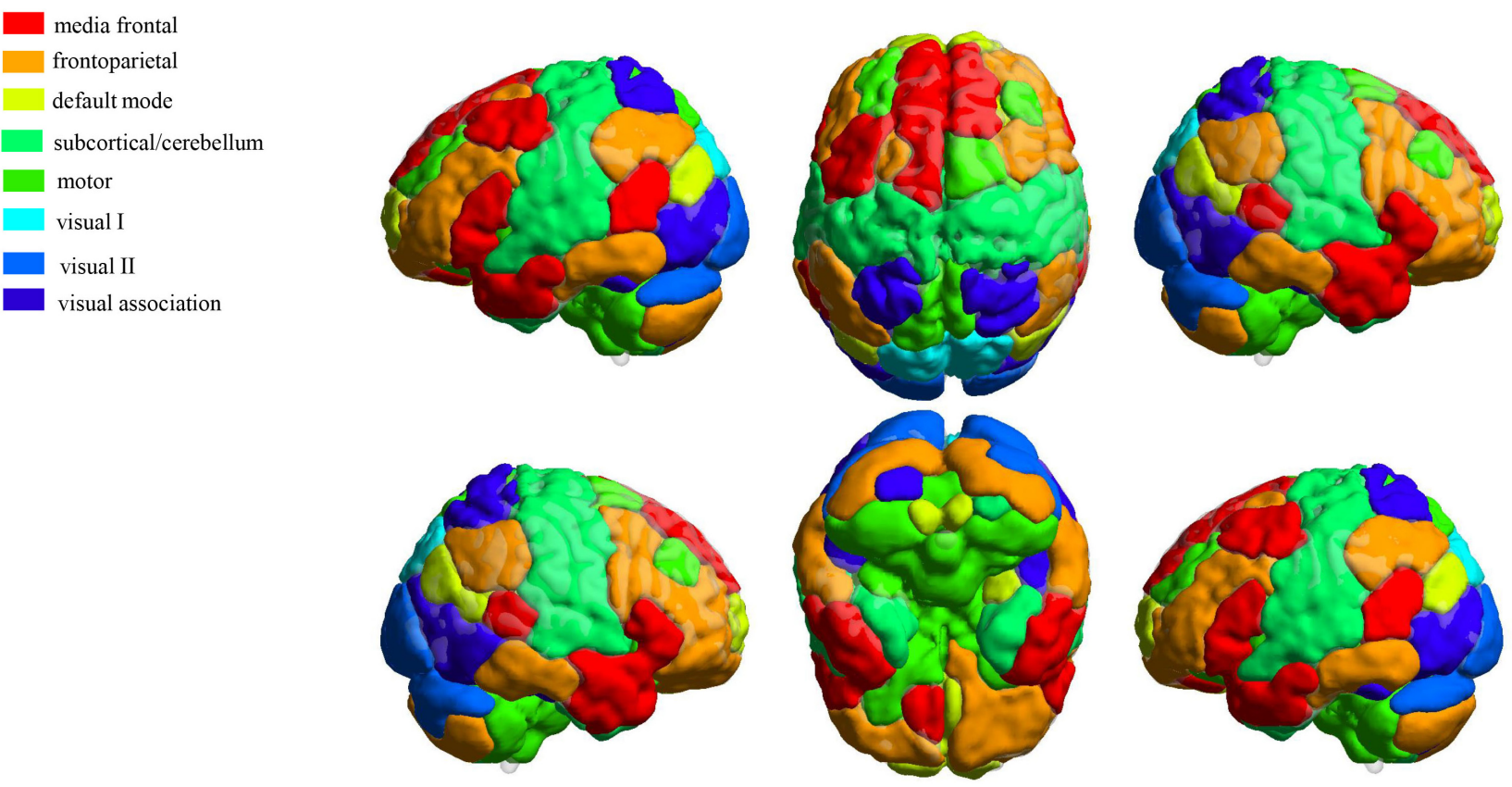

FIGURE 2 | Definition of eight functional networks using a 268-node functional atlas. The figure was generated by using the toolbox BrainNet Viewer (http://www.nitrc.org/projects/bnv/).

TABLE 1 | Demographic and clinical characteristics.

\begin{tabular}{|c|c|c|c|c|c|}
\hline & FESZ patients & CSZ patients & NC & Statistic value & $p$ value \\
\hline $\operatorname{Sex}(F: M)$ & $41: 20$ & $54: 25$ & 110:95 & $x^{2}=3.53$ & 0.03 \\
\hline Age (years) & $32.08 \pm 7.42$ & $33.21 \pm 8.37$ & $32.52 \pm 8.40$ & $F=5.39$ & $<0.05^{\mathrm{a}, \mathrm{b}}$ \\
\hline Education (years) & $10.39 \pm 3.25$ & $11.97 \pm 3.22$ & $12.84 \pm 2.83$ & $F=21.33$ & $<0.05^{a, b}$ \\
\hline PANSS-PScore & $24.02 \pm 4.50$ & $22.47 \pm 5.70$ & - & $T=1.74$ & 0.083 \\
\hline PANSS-NScore & $21.64 \pm 7.70$ & $23.22 \pm 7.29$ & - & $T=-1.24$ & 0.218 \\
\hline PANSS-GScore & $40.31 \pm 8.85$ & $39.54 \pm 10.18$ & - & $T=0.47$ & 0.641 \\
\hline PANSS-TScore & $85.97 \pm 17.49$ & $85.23 \pm 19.44$ & - & $T=0.23$ & 0.816 \\
\hline
\end{tabular}

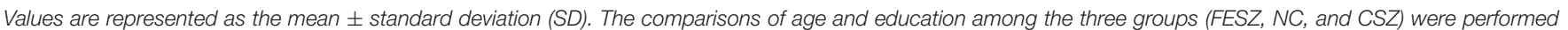

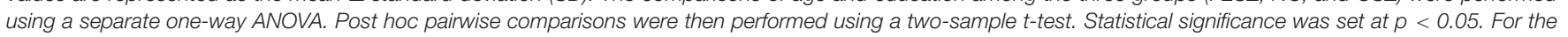
sex distribution among the three groups, the $p$ value was obtained using the $x 2$ test.

a Post hoc paired comparisons showed significant group differences between CSZ vs. NC.

${ }^{b}$ Post hoc paired comparisons showed significant group differences between FESZ vs. NC.

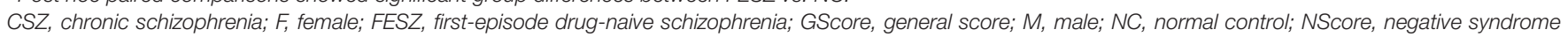
score; PANSS, Positive and Negative Syndrome Scale; PScore, positive syndrome score; TScore, total syndrome score.

and AUC of $92.3 \%(p<0.05)$, as shown in Figure 5 and Supplementary Table 2.

We chose the top $5 \%$ of features ranked by the weights based on the best classification combination (Supplementary Table 4). We found that the ROIs from the GMN were distributed in the subcortical/cerebellum network, frontoparietal network, visual association network, motor network, medial frontal network, visual I network, and visual II network. The ROIs from the WMN were distributed in the subcortical/cerebellum network, frontoparietal network, motor network, visual II network, default mode network, visual I network, visual association network, and medial frontal network (Figure 6).

Overall, the ROIs were mainly distributed in the subcortical/cerebellum network (31.25\%), frontoparietal network (13.75\%) and motor network (15\%), and the proportion of the features from the WMN was greater than the proportion of the features from the GMN in the discrimination FESZ from CSZ patients.

\section{DISCUSSION}

In this study, for the first time, we discriminated SZ patients at different clinical stages using the complex network properties of the GMN, WMN, and FBN. Our main findings were as follows: (1) the SVM algorithm achieved the best performance compared to the other four ML algorithms in both classifications (SZ vs. NC and FESZ vs. CSZ); (2) the 

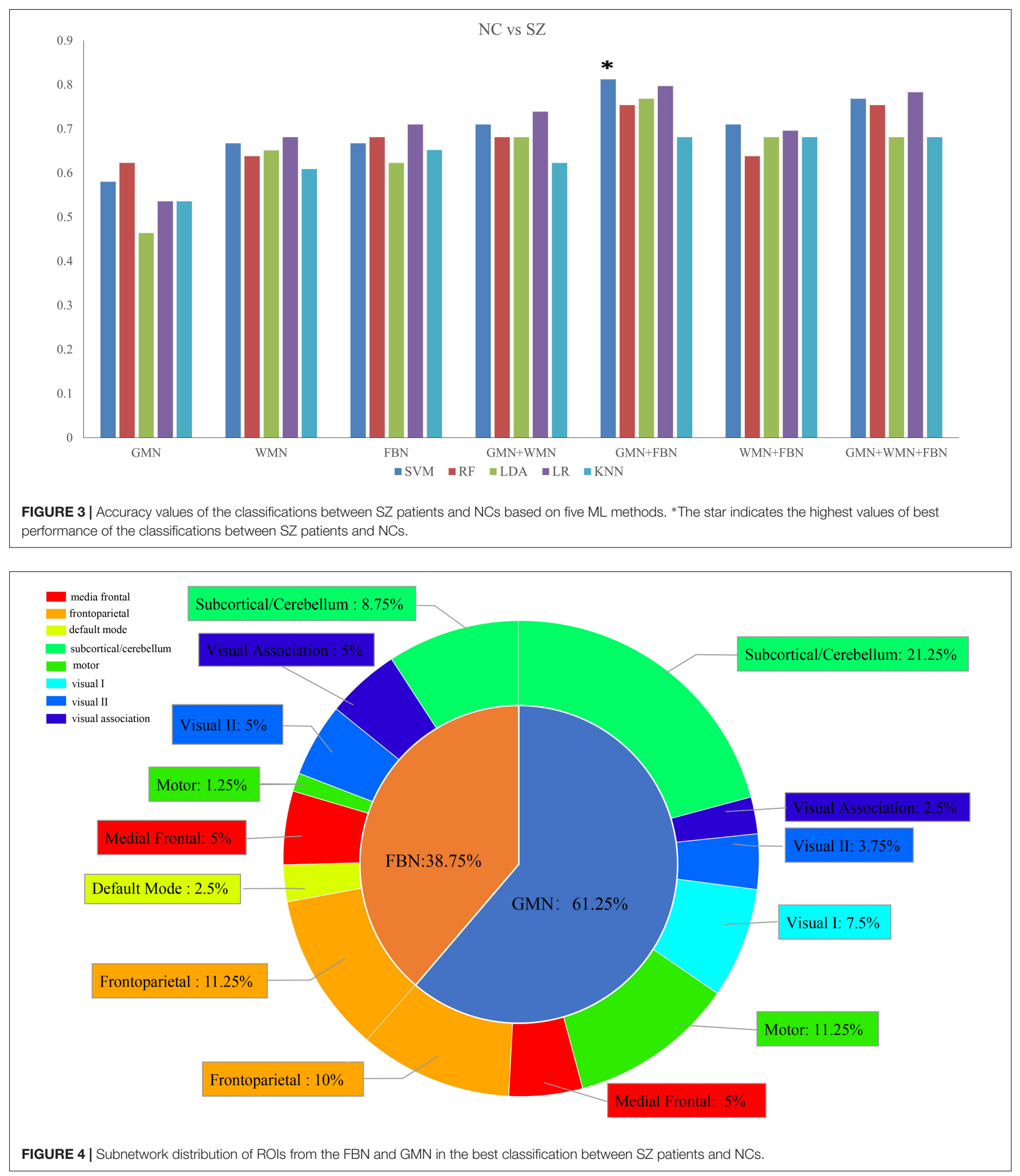

classifier with the input features of GMN and FBN achieved the highest performance in the classification between SZ patients and NCs; the classifier with the input properties of GMN and WMN achieved the highest performance in the classification between FESZ and CSZ patients; and (3) the features of ROIs in both subcortical/cerebellum and frontoparietal networks showed significant importance in both classifications. 


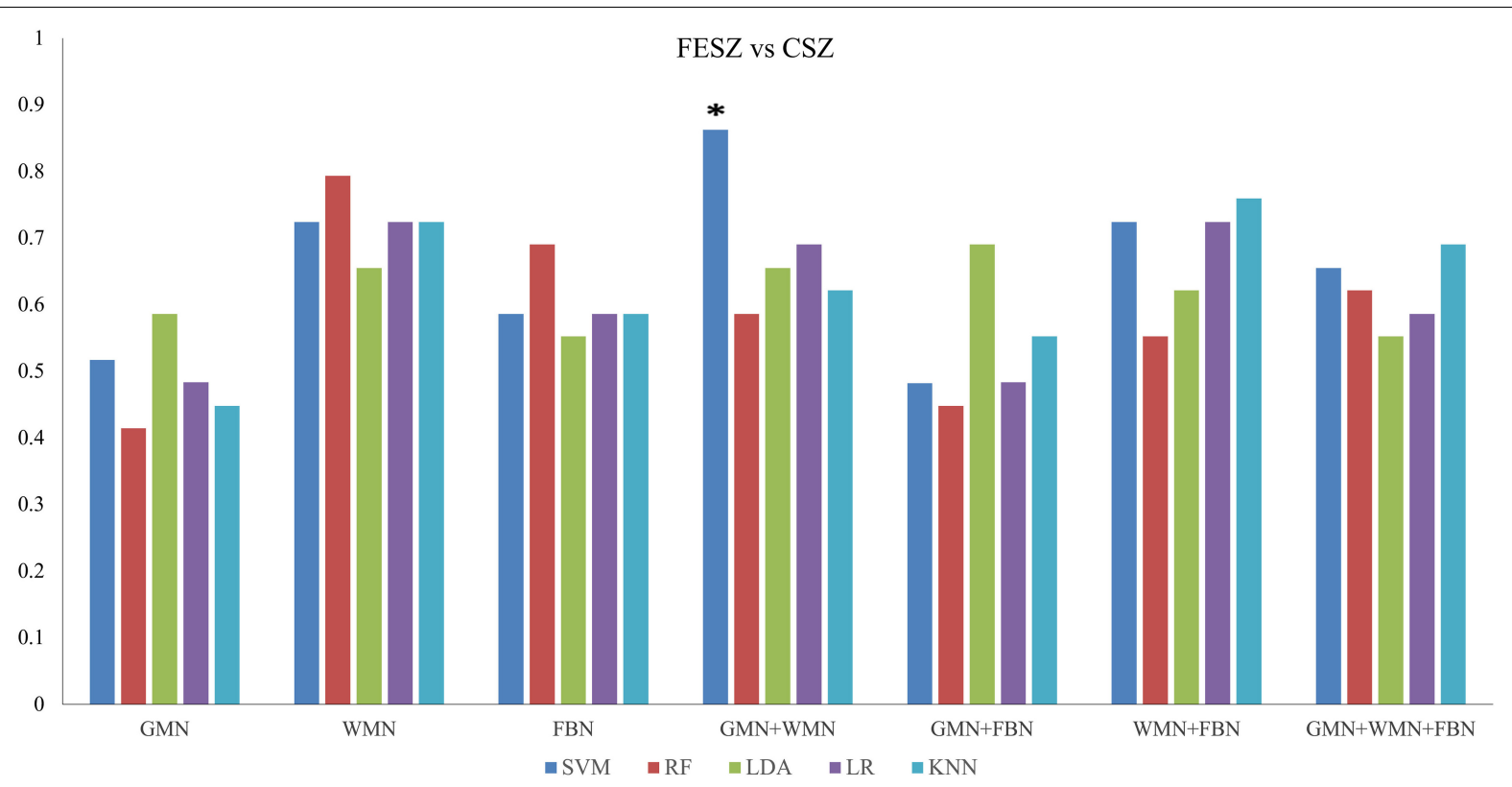

FIGURE 5 | Accuracy values of the classifications between FESZ and CSZ patients based on five ML methods. *The star indicates the highest values of best performance of the classifications between FESZ and CSZ patients.

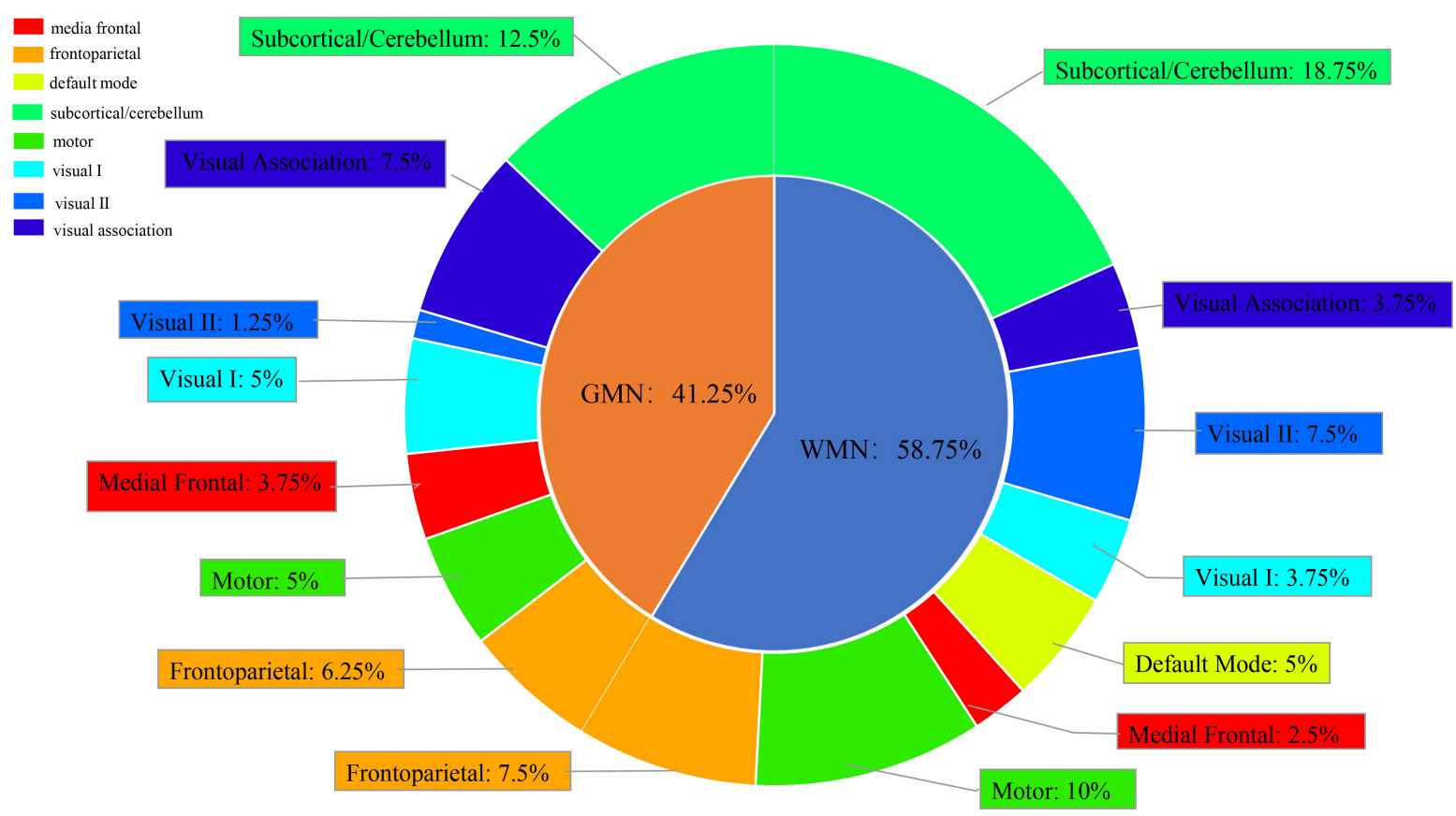

FIGURE 6 | Subnetwork distribution of ROIs from the GMN and WMN in the best classification between FESZ and CSZ patients.

The SVM algorithm, which determines a hyperplane that optimally distinguishes samples into two groups, has been widely used due to its reliable performance (Ding et al., 2015). In this study, our results indicated that the SVM achieved the best performance compared to the other four ML methods (including RF, LR, LDA, and $\mathrm{KNN}$ ) in both classifications, which is consistent with previous studies of SZ (Chin et al., 2018; Rampisela and Rustam, 2018). A previous study discriminated SZ patients from NCs by analyzing multimodal brain imaging data with an SVM classifier and achieved good classification performance with a $91 \%$ accuracy and $100 \%$ prediction rate (Sui et al., 2014). Greenstein et al. (2012) applied the RF 
method to discriminate SZ patients from NCs using 74 anatomic brain MRI subregions and achieved $73.7 \%$ accuracy. Winterburn et al. (2019) performed a critical appraisal of the accuracy of ML methodologies used in SZ patient and NC classifications by comparing three ML methods (including SVM, LR, and LDA), and the highest accuracy achieved was 73.5\% using SVM. Similarly, Li et al. (2015) evaluated the classification performance of four classification methods (including LDA, KNN, SVM, and Gaussian process classifier) on SZ diagnosis and obtained a maximum accuracy of $85.83 \%$ using the SVM. A recent study reviewed five traditional ML algorithms (including SVM, RF, KNN, gradient boosting machine and naive Bayes) frequently used for mental health and indicated that the advantage of SVM was working relatively accurately in general in practice (Cho et al., 2019). Our results confirmed the effectiveness of the SVM algorithm on SZ classification with the features of structural and functional brain networks derived from multimodal MRI data.

The classifier with the input features of the combination of the nodal properties of both GMN and FBN achieved the best performance (accuracy of $81.2 \%$ ) compared to the classifiers using the input features of a single brain network when discriminating SZ from NC. Considerable evidence indicates that SZ is associated with structural as well as functional brain abnormalities (Oh et al., 2017; Li et al., 2020). A recent study revealed that the use of combined structural and functional measures allows the highest accuracy of classification to detect SZ (Lei D. et al., 2020). However, the input features of previous SZ classification studies were derived from structural and functional neuroimaging features (de Filippis et al., 2019; Shi et al., 2021). Our results based on brain network properties further highlight the potential advantages of multimodal features for distinguishing SZ patients from NCs.

Importantly, we also found that the classifier using the nodal properties of both the GMN and WMN achieved the best performance (accuracy of $86.2 \%$ ) when discriminating FESZ from CSZ. The WMN from DTI data played a key role in predicting individuals with FESZ, which is consistent with previous studies (Lee et al., 2013; Samartzis et al., 2014). Friedman et al. (2008) indicated that fractional anisotropy of the white matter in the forceps minor or the genu of the corpus callosum showed striking differences between FESZ and CSZ patients based on DTI findings. White matter abnormalities in SZ as estimated by DTI appear to be present in the early stage of the disorder, most likely reflecting the developmental stage (Kyriakopoulos and Frangou, 2009; Liang et al., 2019). The main objective of our study is to analyze the importance of complex brain networks derived from multi-modal MRI data in the classification of SZ patients at different clinical stages. Our results showed that GMN and FBN played a more important role in discriminating SZ from NCs, whereas GMN and WMN played a more important role in discriminating FESZ from CSZ. Together, these findings may provide new insights into the neuropathology of $\mathrm{SZ}$ at various clinical stages.

In this study, our results demonstrated that the ROIs in the subcortical/cerebellar network and frontoparietal network showed significant importance in SZ classification. A large number of previous studies have indicated that SZ patients showed significantly altered brain connectivity involved in the frontoparietal network, cerebellum network and visual network (Wang et al., 2018; de Filippis et al., 2019). Interestingly, the frontoparietal network and the cerebellum network are among the most commonly implicated networks in SZ patients (Watanabe et al., 2014). The frontoparietal network, which has multiple important hubs in the prefrontal cortex, is involved in executive processing and cognitive control and has been shown to exhibit abnormal activation and connectivity in SZ patients (Minzenberg et al., 2009; Cole et al., 2013; Tu et al., 2013). The cerebellum network, which is featured in the influential 'cognitive dysmetria' hypothesis of SZ patients (Andreasen et al., 1998), has been found to be the most important network in the functional connectivity of SZ patients according to the extended maximal information coefficient (Su et al., 2013). Moreover, previous studies based on deep learning algorithms have discovered that cortical-striatal-cerebellar functional connectivity features exhibit great weights in the classification of SZ (Zeng et al., 2018; Qureshi et al., 2019). We also found that the ROIs in these two networks showed key importance in discriminating FESZ and CSZ patients. A meta-analysis of 25 articles based on cerebellar structural and functional abnormalities in FESZ patients hypothesized that the changes in both structural and functional aspects might reflect a common pathophysiology of the cerebellum in SZ patients (Ding et al., 2019). Lui et al. (2010) reported that 6 weeks of antipsychotic treatment increased brain synchronous activity in the frontal and parietal regions in FESZ patients, which might contribute to the importance of the frontoparietal network in discriminating FESZ and CSZ patients. In addition, the ROIs in the motor network also showed significant importance in discriminating FESZ patients from CSZ patients but not in SZ patients from NCs. It was reported that the functional connectivity in the sensory-motor network could reflect the hypothesized "neurotoxic effect" of FESZ patients (Zhang et al., 2019), demonstrating that the motor network deserves more attention in the search for neuroimaging markers for evaluating neural impairment in SZ.

Interestingly, we found that the $\mathrm{BC}$ of network topological features has more importance than DC and NE to the classifier for both contrast groups. The $\mathrm{BC}$ is a ratio of the number of all shortest paths between any two nodes in the network that travel through an index node, providing an indication of how topologically central the role of node is in overall network communication (van den Heuvel and Fornito, 2014). Previous brain network studies have indicated that BC in some brain regions is decreased (van den Heuvel and Hulshoff Pol, 2010; Zhang et al., 2012). Our study may further confirm the importance of the $\mathrm{BC}$ of network topological features in identifying SZ.

\section{LIMITATIONS OF THE METHODOLOGY}

Possible study limitations should be considered. First, only three nodal features (BC, DC, and $\mathrm{NC}$ ) were combined in the present study. Additional network measures, such as clustering coefficient, path length and global efficiency, can be used and may improve classification performance. Second, we compared 
the performance of five ML methods using only RFE as the dimensionality reduction algorithm. Previous studies of SZ patients have shown different classification performances using different dimensionality reduction algorithms (Li et al., 2015; Winterburn et al., 2019). Third, we have used different brain atlases in the discriminative analysis of SZ patients based on neuroimaging features and revealed that the 268-node functional atlas outperformed the other two brain atlases (Zang et al., 2021). However, it is necessary to compare the effects among different combinations of dimensionality reduction algorithms and brain atlases from the perspective of brain network properties in future studies. Fourth, the classification performance did not always benefit from more modalities in either classification. Previous studies have also shown that including more features does not necessarily yield positive effects due to feature redundancy. However, a recent study proposed a safe classifier that could address this issue to some extent (Hou et al., 2019). It is important to study whether safe classification would affect the discriminative results based on multimodal brain networks in future studies.

\section{CONCLUSION}

We constructed GMNs, WMNs and FBNs from sMRI, DTI and fMRI data, respectively and then discriminated SZ patients at different clinical stages using different combinations of nodal properties based on five ML methods. Our results indicated the best performance of the SVM algorithm in SZ classifications and highlighted the potential advantages of multimodal network properties in identifying SZ patients at different stages. Furthermore, the ROIs in the subcortical/cerebellum network and frontoparietal network showed significant importance in both classifications (NC vs. SZ, FESZ vs. CSZ). Our findings may bring new insights into the understanding of the neuropathology of SZ from the perspective of network properties.

\section{DATA AVAILABILITY STATEMENT}

The raw data supporting the conclusions of this article will be made available by the authors without undue reservation.

\section{REFERENCES}

Adriano, F., Caltagirone, C., and Spalletta, G. (2012). Hippocampal volume reduction in first-episode and chronic schizophrenia. Neuroscientist 18, 180200. doi: $10.1177 / 1073858410395147$

Adriano, F., Spoletini, I., Caltagirone, C., and Spalletta, G. (2010). Updated metaanalyses reveal thalamus volume reduction in patients with first-episode and chronic schizophrenia. Schizophr. Res. 123, 1-14. doi: 10.1016/j.schres.2010.07. 007

Andreasen, N. C., Paradiso, S., and O'Leary, D. S. (1998). "Cognitive dysmetria” as an integrative theory of schizophrenia: a dysfunction in cortical-subcorticalcerebellar circuitry? Schizophr. Bull. 24, 203-218. doi: 10.1093/oxfordjournals. schbul.a033321

\section{ETHICS STATEMENT}

The studies involving human participants were reviewed and approved by Ethics Committees of the Affiliated Brain Hospital of Guangzhou Medical University. The patients/participants provided their written informed consent to participate in this study.

\section{AUTHOR CONTRIBUTIONS}

All authors contributed to data analysis, drafted and critically revised the manuscript, gave final approval of the version to be published, and agreed to be accountable for all aspects of the work.

\section{FUNDING}

This work was supported by the National Key Research and Development Program of China (2020YFC2004300, 2020YFC2004301, 2019YFC0118800, 2019YFC0118802, 2019YFC0118804, 2019YFC0118805, 2021YFC2009400, and 2021YFC2009404), National Natural Science Foundation of China (31771074, 81802230, and 72174082), Guangdong Basic and Applied Basic Research Foundation Outstanding Youth Project (2021B1515020064), Key Research and Development Program of Guangdong (2018B030335001, 2020B0101130020, and 2020B0404010002), Science and Technology Program of Guangzhou (201807010064, 201803010100, 201903010032, and 202103000032), Key Laboratory Program of Guangdong Provincial Education Department (2020KSYS001), Open Fund of the Guangdong Provincial Key Laboratory of Physical Activity and Health Promotion (2021B1212040014), and Science Foundation of Guangzhou Xinhua University (2018YB002 and 2020YQYJ05).

\section{SUPPLEMENTARY MATERIAL}

The Supplementary Material for this article can be found online at: https://www.frontiersin.org/articles/10.3389/fnins. 2021.785595/full\#supplementary-material

Antonova, E., Kumari, V., Morris, R., Halari, R., Anilkumar, A., Mehrotra, R., et al. (2005). The relationship of structural alterations to cognitive deficits in schizophrenia: a voxel-based morphometry study. Biol. Psychiatry 58, 457-467. doi: 10.1016/j.biopsych.2005.04.036

Arbabshirani, M. R., Kiehl, K. A., Pearlson, G. D., and Calhoun, V. D. (2013). Classification of schizophrenia patients based on resting-state functional network connectivity. Front. Neurosci. 7:133. doi: 10.3389/fnins.2013.00133

Ardekani, B. A., Tabesh, A., Sevy, S., Robinson, D. G., Bilder, R. M., and Szeszko, P. R. (2011). Diffusion tensor imaging reliably differentiates patients with schizophrenia from healthy volunteers. Hum. Brain Mapp. 32, 1-9. doi: 10. 1002/hbm.20995

Bassett, D. S., Bullmore, E., Verchinski, B. A., Mattay, V. S., Weinberger, D. R., and Meyer-Lindenberg, A. (2008). Hierarchical organization of human cortical 
networks in health and schizophrenia. J. Neurosci. 28, 9239-9248. doi: 10.1523/ JNEUROSCI.1929-08.2008

Breiman, L. (1996). Bagging predictors. Mach. Learn. 2, 123-140.

Cai, X. L., Xie, D. J., Madsen, K. H., Wang, Y. M., Bögemann, S. A., Cheung, E. F. C., et al. (2020). Generalizability of machine learning for classification of schizophrenia based on resting-state functional MRI data. Hum. Brain Mapp. 41, 172-184. doi: 10.1002/hbm.24797

Cao, B., Cho, R. Y., Chen, D., Xiu, M., Wang, L., Soares, J. C., et al. (2020). Treatment response prediction and individualized identification of first-episode drug-naïve schizophrenia using brain functional connectivity. Mol. Psychiatry 25, 906-913. doi: 10.1038/s41380-018-0106-5

Chen, C., Wang, H. L., Wu, S. H., Huang, H., Zou, J. L., Chen, J., et al. (2015). Abnormal degree centrality of bilateral putamen and left superior frontal gyrus in schizophrenia with auditory hallucinations: a resting-state functional magnetic resonance imaging study. Chin. Med. J. (Engl.) 128, 3178-3184. doi: 10.4103/0366-6999.170269

Chin, R., You, A. X., Meng, F., Zhou, J., and Sim, K. (2018). Recognition of schizophrenia with regularized support vector machine and sequential region of interest selection using structural magnetic resonance imaging. Sci. Rep. 8:13858. doi: 10.1038/s41598-018-32290-9

Cho, G., Yim, J., Choi, Y., Ko, J., and Lee, S. (2019). Review of machine learning algorithms for diagnosing mental illness. Psychiatry Investig. 16, 262-269. doi: 10.30773/pi.2018.12.21.2

Cole, M. W., Reynolds, J. R., Power, J. D., Repovs, G., Anticevic, A., and Braver, T. S. (2013). Multi-task connectivity reveals flexible hubs for adaptive task control. Nat. Neurosci. 16, 1348-1355. doi: 10.1038/nn.3470

Collin, G., Kahn, R. S., de Reus, M. A., Cahn, W., and van den Heuvel, M. P. (2014). Impaired rich club connectivity in unaffected siblings of schizophrenia patients. Schizophr. Bull. 40, 438-448. doi: 10.1093/schbul/sbt162

Cortes, C. V. V. (1995). Support-vectorNetworks. Mach. Learn. 20, 273-297.

Cui, Z., Zhong, S., Xu, P., He, Y., and Gong, G. (2013). PANDA: a pipeline toolbox for analyzing brain diffusion images. Front. Hum. Neurosci. 7:42. doi: 10.3389/ fnhum.2013.00042

de Filippis, R., Carbone, E. A., Gaetano, R., Bruni, A., Pugliese, V., Segura-Garcia, C., et al. (2019). Machine learning techniques in a structural and functional MRI diagnostic approach in schizophrenia: a systematic review. Neuropsychiatr. Dis. Treat. 15, 1605-1627. doi: 10.2147/NDT.S202418

Ding, X., Yang, Y., Stein, E. A., and Ross, T. J. (2015). Multivariate classification of smokers and nonsmokers using SVM-RFE on structural MRI images. Hum. Brain Mapp. 36, 4869-4879. doi: 10.1002/hbm.22956

Ding, Y., Ou, Y., Pan, P., Shan, X., Chen, J., Liu, F., et al. (2019). Cerebellar structural and functional abnormalities in first-episode and drug-naive patients with schizophrenia: a meta-analysis. Psychiatry Res. Neuroimaging 283, 24-33. doi: 10.1016/j.pscychresns.2018.11.009

Drakesmith, M., Caeyenberghs, K., Dutt, A., Zammit, S., Evans, C. J., Reichenberg, A., et al. (2015). Schizophrenia-like topological changes in the structural connectome of individuals with subclinical psychotic experiences. Hum. Brain Mapp. 36, 2629-2643. doi: 10.1002/hbm.22796

Erdeniz, B., Serin, E., İbadi, Y., and Taş, C. (2017). Decreased functional connectivity in schizophrenia: the relationship between social functioning, social cognition and graph theoretical network measures. Psychiatry Res. Neuroimaging 270, 22-31. doi: 10.1016/j.pscychresns.2017.09.011

Finn, E. S., Shen, X., Scheinost, D., Rosenberg, M. D., Huang, J., Chun, M. M., et al. (2015). Functional connectome fingerprinting: identifying individuals using patterns of brain connectivity. Nat.e Neurosci. 18, 1664-1671. doi: 10.1038/nn. 4135

Fisher, R. A. (1936). The use of multiple measurements in taxonomic problems. Ann. Eugen. 2, 179-188.

Friedman, J. I., Tang, C., Carpenter, D., Buchsbaum, M., Schmeidler, J., Flanagan, L., et al. (2008). Diffusion tensor imaging findings in first-episode and chronic schizophrenia patients. Am. J. Psychiatry 165, 1024-1032. doi: 10.1176/appi.ajp. 2008.07101640

Gong, G., He, Y., Concha, L., Lebel, C., Gross, D. W., Evans, A. C., et al. (2009). Mapping anatomical connectivity patterns of human cerebral cortex using in vivo diffusion tensor imaging tractography. Cereb. Cortex 19, 524-536. doi: 10.1093/cercor/bhn102

Good, C. D., Johnsrude, I., Ashburner, J., Henson, R. N. A., Friston, K. J., and Frackowiak, R. S. J. (2001). Cerebral asymmetry and the effects of sex and handedness on brain structure: a voxel-based morphometric analysis of 465 normal adult human brains. NeuroImage 14, 685-700. doi: 10.1006/nimg.2001. 0857

Greenstein, D., Malley, J. D., Weisinger, B., Clasen, L., and Gogtay, N. (2012). Using multivariate machine learning methods and structural MRI to classify childhood onset schizophrenia and healthy controls. Front. Psychiatry 3:53. doi: 10.3389/fpsyt.2012.00053

Griffa, A., Baumann, P. S., Ferrari, C., Do, K. Q., Conus, P., Thiran, J., et al. (2015). Characterizing the connectome in schizophrenia with diffusion spectrum imaging. Hum. Brain Mapp. 36, 354-366. doi: 10.1002/hbm.22633

Guo, Y., Qiu, J., and Lu, W. (2020). Support vector machine-based schizophrenia classification using morphological information from amygdaloid and hippocampal subregions. Brain Sci. 10:562. doi: 10.3390/brainsci10080562

Hou, C., Zeng, L., and Hu, D. (2019). Safe classification with augmented features. IEEE Trans. Pattern Anal. Mach. Intell. 41, 2176-2192. doi: 10.1109/TPAMI. 2018.2849378

Ingalhalikar, M., Kanterakis, S., Gur, R., Roberts, T. P., and Verma, R. (2010). DTI based diagnostic prediction of a disease via pattern classification. Med. Image Comput. Comput. Assist. Interv. 13(Pt 1), 558-565. doi: 10.1007/978-3-64215705-9_68

Kay, S. R., Fiszbein, A., and Opler, L. A. (1987). The positive and negative syndrome scale (PANSS) for schizophrenia. Schizophr. Bull. 13, 261-276.

Ke, P., Xiong, D., Li, J., Pan, Z., Zhou, J., Li, S., et al. (2021). An integrated machine learning framework for a discriminative analysis of schizophrenia using multi-biological data. Sci. Rep. 11:14636. doi: 10.1038/s41598-021-940079

Koch, S. P., Hägele, C., Haynes, J., Heinz, A., Schlagenhauf, F., and Sterzer, P. (2015). Diagnostic classification of schizophrenia patients on the basis of regional reward-related fMRI signal patterns. PLoS One 10:e119089. doi: 10. 1371/journal.pone.0119089

Kong, L., Huang, Y., Lei, B., Ke, P., Li, H., Zhou, J., et al. (2021). Divergent alterations of structural-functional connectivity couplings in first-episode and chronic schizophrenia patients. Neuroscience 460, 1-12. doi: 10.1016/j. neuroscience.2021.02.008

Kong, X., Wang, X., Huang, L., Pu, Y., Yang, Z., Dang, X., et al. (2014). Measuring individual morphological relationship of cortical regions. J. Neurosci. Methods 237, 103-107. doi: 10.1016/j.jneumeth.2014.09.003

Kuroki, N. (2006). Middle and inferior temporal gyrus gray matter volume abnormalities in first-episode schizophrenia: an MRI study. Am. J. Psychiatry 163:2103. doi: 10.1176/ajp.2006.163.12.2103

Kyriakopoulos, M., and Frangou, S. (2009). Recent diffusion tensor imaging findings in early stages of schizophrenia. Curr. Opin. Psychiatry 22, 168-176. doi: 10.1097/YCO.0b013e328325aa23

Lee, S., Kubicki, M., Asami, T., Seidman, L. J., Goldstein, J. M., Mesholam-Gately, R. I., et al. (2013). Extensive white matter abnormalities in patients with firstepisode schizophrenia: A diffusion tensor imaging (DTI) study. Schizophr. Res. 143, 231-238.

Lei, B., Wu, F., Zhou, J., Xiong, D., Wang, K., Kong, L., et al. (2020). NEUROLEARN: a solution for collaborative pattern analysis of neuroimaging data. Neuroinformatics 19, 79-91. doi: 10.1007/s12021-020-09468-6

Lei, D., Pinaya, W. H. L., Young, J., Amelsvoort, T., Marcelis, M., Donohoe, G., et al. (2020). Integrating machining learning and multimodal neuroimaging to detect schizophrenia at the level of the individual. Hum. Brain Mapp. 41, 1119-1135. doi: 10.1002/hbm.24863

Li, A., Zalesky, A., Yue, W., Howes, O., Yan, H., Liu, Y., et al. (2020). A neuroimaging biomarker for striatal dysfunction in schizophrenia. Nat. Med. 26, 558-565. doi: 10.1038/s41591-020-0793-8

Li, Z., Wang, Y., Quan, W., Wu, T., and Lv, B. (2015). Evaluation of different classification methods for the diagnosis of schizophrenia based on functional near-infrared spectroscopy. J. Neurosci. Methods 241, 101-110. doi: 10.1016/j. jneumeth.2014.12.020

Liang, S., Li, Y., Zhang, Z., Kong, X., Wang, Q., Deng, W., et al. (2019). Classification of first-episode schizophrenia using multimodal brain features: a combined structural and diffusion imaging study. Schizophr. Bull. 45, 591-599. doi: 10.1093/schbul/sby091

Lin, X., Li, C., Zhang, Y., Su, B., Fan, M., and Wei, H. (2017). Selecting feature subsets based on SVM-RFE and the overlapping ratio with applications in bioinformatics. Molecules 23:52. doi: 10.3390/molecules23010052 
Liu, Y., Wang, H., Duan, Y., Huang, J., Ren, Z., Ye, J., et al. (2017). Functional brain network alterations in clinically isolated syndrome and multiple sclerosis: a graph-based connectome study. Radiology 282, 534-541. doi: 10.1148/radiol. 2016152843

Lu, X. B., Zhang, Y., Yang, D. Y., Yang, Y. Z., Wu, F. C., Ning, Y. P., et al. (2018). Analysis of first-episode and chronic schizophrenia using multi-modal magnetic resonance imaging. Eur. Rev. Med. Pharmacol. Sci. 22, 6422-6435. doi: 10.26355/eurrev_201810_16055

Lu, X., Yang, Y., Wu, F., Gao, M., Xu, Y., Zhang, Y., et al. (2016). Discriminative analysis of schizophrenia using support vector machine and recursive feature elimination on structural MRI images. Medicine 95:e3973.

Lui, S., Li, T., Deng, W., Jiang, L., Wu, Q., Tang, H., et al. (2010). Short-term effects of antipsychotic treatment on cerebral function in drug-naive firstepisode schizophrenia revealed by "resting state" functional magnetic resonance imaging. Arch. Gen. Psychiatry 67:783. doi: 10.1001/archgenpsychiatry.20 10.84

Minzenberg, M. J., Laird, A. R., Thelen, S., Carter, C. S., and Glahn, D. C. (2009). Meta-analysis of 41 functional neuroimaging studies of executive function in schizophrenia. Arch. Gen. Psychiatry 66:811. doi: 10.1001/archgenpsychiatry. 2009.91

Moncrieff, J., and Leo, J. (2010). A systematic review of the effects of antipsychotic drugs on brain volume. Psychol. Med. 40, 1409-1422. doi: 10. 1017/S0033291709992297

Oh, J., Chun, J., Kim, E., Park, H., Lee, B., and Kim, J. (2017). Aberrant neural networks for the recognition memory of socially relevant information in patients with schizophrenia. Brain Behav. 7:e602. doi: 10.1002/brb3.602

Oh, J., Oh, B., Lee, K., Chae, J., and Yun, K. (2020). Identifying schizophrenia using structural MRI With a deep learning algorithm. Front. Psychiatry 11:16. doi: 10.3389/fpsyt.2020.00016

Pagsberg, A. K., Baaré, W. F. C., Raabjerg Christensen, A. M., Fagerlund, B., Hansen, M. B., Labianca, J., et al. (2007). Structural brain abnormalities in early onset first-episode psychosis. J. Neural Transm. 114, 489-498. doi: 10.1007/ s00702-006-0573-8

Palaniyappan, L., Park, B., Balain, V., Dangi, R., and Liddle, P. (2015). Abnormalities in structural covariance of cortical gyrification in schizophrenia. Brain Struct. Funct. 220, 2059-2071. doi: 10.1007/s00429-014-0772-2

Peng, C. J., Lee, K. L., and Ingersoll, G. M. (2002). An introduction to logistic regression analysis and reporting. J. Educ. Res. 96, 3-14.

Qureshi, M. N. I., Oh, J., and Lee, B. (2019). 3D-CNN based discrimination of schizophrenia using resting-state fMRI. Artific. Intell. Med. 98, 10-17. doi: 10.1016/j.artmed.2019.06.003

Rampisela, T. V., and Rustam, Z. (2018). Classification of schizophrenia data using support vector machine (SVM). J. Phys. Conf. Ser. 1108:12044.

Ren, W., Lui, S., Deng, W., Li, F., Li, M., Huang, X., et al. (2013). Anatomical and functional brain abnormalities in drug-naive first-episode schizophrenia. Am. J. Psychiatry 170, 1308-1316. doi: 10.1176/appi.ajp.2013.12091148

Samartzis, L., Dima, D., Fusar-Poli, P., and Kyriakopoulos, M. (2014). White matter alterations in early stages of schizophrenia: a systematic review of diffusion tensor imaging studies. J. Neuroimaging 24, 101-110. doi: 10.1111/j.1552-6569. 2012.00779.x

Shen, X., Tokoglu, F., Papademetris, X., and Constable, R. T. (2013). Groupwise whole-brain parcellation from resting-state fMRI data for network node identification. NeuroImage 82, 403-415. doi: 10.1016/j.neuroimage.2013.05.081

Shi, D., Li, Y., Zhang, H., Yao, X., Wang, S., Wang, G., et al. (2021). Machine learning of schizophrenia detection with structural and functional neuroimaging. Dis. Mark. 2021, 1-12. doi: 10.1155/2021/9963824

Skåtun, K. C., Kaufmann, T., Doan, N. T., Alnæs, D., Córdova-Palomera, A., Jönsson, E. G., et al. (2017). Consistent functional connectivity alterations in schizophrenia spectrum disorder: a multisite study. Schizophr. Bull. 43, 914-924. doi: 10.1093/schbul/sbw145

Steardo, L., Carbone, E. A., de Filippis, R., Pisanu, C., Segura-Garcia, C., Squassina, A., et al. (2020). Application of support vector machine on fMRI data as biomarkers in schizophrenia diagnosis: a systematic review. Front. Psychiatry 11:588. doi: 10.3389/fpsyt.2020.00588

Su, L., Wang, L., Shen, H., Feng, G., and Hu, D. (2013). Discriminative analysis of non-linear brain connectivity in schizophrenia: an fMRI Study. Front. Hum. Neurosci. 7:702. doi: 10.3389/fnhum.2013. 00702
Sui, J., Castro, E., He, H., Bridwell, D., Du, Y., Pearlson, G. D., et al. (2014). Combination of FMRI-SMRI-EEG data improves discrimination of schizophrenia patients by ensemble feature selection. Annu. Int. Conf. IEEE Eng. Med. Biol. Soc. 2014, 3889-3892. doi: 10.1109/EMBC.2014.6944473

Sun, Y., Chen, Y., Collinson, S. L., Bezerianos, A., and Sim, K. (2015). Reduced hemispheric asymmetry of brain anatomical networks is linked to schizophrenia: a connectome study. Cereb. Cortex 255, 1-14. doi: 10.1093/ cercor/bhv255

Talpalaru, A., Bhagwat, N., Devenyi, G. A., Lepage, M., and Chakravarty, M. M. (2019). Identifying schizophrenia subgroups using clustering and supervised learning. Schizophr. Res. 214, 51-59. doi: 10.1016/j.schres.2019.05.044

Torres, U. S., Duran, F. L. S., Schaufelberger, M. S., Crippa, J. A. S., Louzã, M. R., Sallet, P. C., et al. (2016). Patterns of regional gray matter loss at different stages of schizophrenia: a multisite, cross-sectional VBM study in first-episode and chronic illness. NeuroImage Clin. 12, 1-15. doi: 10.1016/j.nicl.2016.06.002

Tu, P., Lee, Y., Chen, Y., Li, C., and Su, T. (2013). Schizophrenia and the brain's control network: aberrant within- and between-network connectivity of the frontoparietal network in schizophrenia. Schizophr. Res. 147, 339-347. doi: 10.1016/j.schres.2013.04.011

Turner, J. A. (2013). A multi-site resting state fMRI study on the amplitude of low frequency fluctuations in schizophrenia. Front. Neurosci. 7:137. doi: 10.3389/ fnins.2013.00137

van den Heuvel, M. P., and Fornito, A. (2014). Brain networks in schizophrenia. Neuropsychol. Rev. 24, 32-48.

van den Heuvel, M. P., and Hulshoff Pol, H. E. (2010). Exploring the brain network: a review on resting-state fMRI functional connectivity. Eur. Neuropsychopharmacol. 20, 519-534. doi: 10.1016/j.euroneuro.2010.03.008

van den Heuvel, M. P., Mandl, R. C. W., Stam, C. J., Kahn, R. S., and Hulshoff Pol, H. E. (2010). Aberrant frontal and temporal complex network structure in schizophrenia: a graph theoretical analysis. J. Neurosci. 30, 15915-15926. doi: 10.1523/JNEUROSCI.2874-10.2010

van den Heuvel, M. P., Sporns, O., Collin, G., Scheewe, T., Mandl, R. C. W., Cahn, W., et al. (2013). Abnormal rich club organization and functional brain dynamics in schizophrenia. JAMA Psychiatry 70, 783-792. doi: 10.1001/ jamapsychiatry.2013.1328

van Tol, M., van der Meer, L., Bruggeman, R., Modinos, G., Knegtering, H., and Aleman, A. (2014). Voxel-based gray and white matter morphometry correlates of hallucinations in schizophrenia: the superior temporal gyrus does not stand alone. NeuroImage Clin. 4, 249-257. doi: 10.1016/j.nicl.2013.12.008

Wang, H., Jin, X., Zhang, Y., and Wang, J. (2016). Single-subject morphological brain networks: connectivity mapping, topological characterization and testretest reliability. Brain Behav. 6:e00448. doi: 10.1002/brb3.448

Wang, J., Wang, X., Xia, M., Liao, X., Evans, A., and He, Y. (2015). GRETNA: a graph theoretical network analysis toolbox for imaging connectomics. Front. Hum. Neurosci. 9:386. doi: 10.3389/fnhum.2015.00386

Wang, Q., Su, T., Zhou, Y., Chou, K., Chen, I., Jiang, T., et al. (2012). Anatomical insights into disrupted small-world networks in schizophrenia. NeuroImage 59, 1085-1093. doi: 10.1016/j.neuroimage.2011.09.035

Wang, S., Zhan, Y., Zhang, Y., Lyu, L., Lyu, H., Wang, G., et al. (2018). Abnormal long- and short-range functional connectivity in adolescent-onset schizophrenia patients: a resting-state fMRI study. Prog. Neuropsychopharmacol. Biol. Psychiatry 81, 445-451. doi: 10.1016/j.pnpbp.2017.08.012

Watanabe, T., Kessler, D., Scott, C., Angstadt, M., and Sripada, C. (2014). Disease prediction based on functional connectomes using a scalable and spatiallyinformed support vector machine. NeuroImage 96, 183-202. doi: 10.1016/j. neuroimage.2014.03.067

Winterburn, J. L., Voineskos, A. N., Devenyi, G. A., Plitman, E., de la FuenteSandoval, C., Bhagwat, N., et al. (2019). Can we accurately classify schizophrenia patients from healthy controls using magnetic resonance imaging and machine learning? A multi-method and multi-dataset study. Schizophr. Res. 214, 3-10. doi: 10.1016/j.schres.2017.11.038

Wu, F., Zhang, Y., Yang, Y., Lu, X., Fang, Z., Huang, J., et al. (2018). Structural and functional brain abnormalities in drug-naive, first-episode, and chronic patients with schizophrenia: a multimodal MRI study. Neuropsychiatr. Dis. Treat. 14, 2889-2904. doi: 10.2147/NDT.S174356

Xiao, Y., Yan, Z., Zhao, Y., Tao, B., Sun, H., Li, F., et al. (2017). Support vector machine-based classification of first episode drug-naïve schizophrenia patients 
and healthy controls using structural MRI. Schizophr. Res. 214, 11-17. doi: 10.1016/j.schres.2017.11.037

Yamasue, H., Iwanami, A., Hirayasu, Y., Yamada, H., Abe, O., Kuroki, N., et al. (2004). Localized volume reduction in prefrontal, temporolimbic, and paralimbic regions in schizophrenia: an MRI parcellation study. Psychiatry Res. 131, 195-207. doi: 10.1016/j.pscychresns.2004.05.004

Yan, C., Wang, X., Zuo, X., and Zang, Y. (2016). DPABI: data processing \& analysis for (resting-state) brain imaging. Neuroinformatics 14, 339-351.

Zang, J., Huang, Y., Kong, L., Lei, B., Ke, P., Li, H., et al. (2021). Effects of brain atlases and machine learning methods on the discrimination of schizophrenia patients: a multimodal MRI study. Front. Neurosci. 15:697168. doi: 10.3389/ fnins.2021.697168

Zeng, L., Wang, H., Hu, P., Yang, B., Pu, W., Shen, H., et al. (2018). Multi-site diagnostic classification of schizophrenia using discriminant deep learning with functional connectivity MRI. EBioMedicine 30, 74-85. doi: 10.1016/j.ebiom. 2018.03.017

Zhang, Y., Lin, L., Lin, C., Zhou, Y., Chou, K., Lo, C., et al. (2012). Abnormal topological organization of structural brain networks in schizophrenia. Schizophr. Res. 141, 109-118. doi: 10.1016/j.schres.2012.08.021

Zhang, Y., Xu, L., Hu, Y., Wu, J., Li, C., Wang, J., et al. (2019). Functional connectivity between sensory-motor subnetworks reflects the duration of untreated psychosis and predicts treatment outcome of first-episode drug-naïve schizophrenia. Biol. Psychiatry Cogn. Neurosci. Neuroimaging 4, 697-705. doi: 10.1016/j.bpsc.2019.04.002
Zhang, Z. (2016). Introduction to machine learning: k-nearest neighbors. Ann. Transl. Med. 4:218. doi: 10.21037/atm.2016.03.37

Zhou, Y., Shu, N., Liu, Y., Song, M., Hao, Y., Liu, H., et al. (2007). Altered restingstate functional connectivity and anatomical connectivity of hippocampus in schizophrenia. Schizophr. Res. 100, 120-132. doi: 10.1016/j.schres.2007. 11.039

Conflict of Interest: The authors declare that the research was conducted in the absence of any commercial or financial relationships that could be construed as a potential conflict of interest.

Publisher's Note: All claims expressed in this article are solely those of the authors and do not necessarily represent those of their affiliated organizations, or those of the publisher, the editors and the reviewers. Any product that may be evaluated in this article, or claim that may be made by its manufacturer, is not guaranteed or endorsed by the publisher.

Copyright () 2022 Wang, Ke, Zang, Wu and Wu. This is an open-access article distributed under the terms of the Creative Commons Attribution License (CC BY). The use, distribution or reproduction in other forums is permitted, provided the original author(s) and the copyright owner(s) are credited and that the original publication in this journal is cited, in accordance with accepted academic practice. No use, distribution or reproduction is permitted which does not comply with these terms. 